\title{
COTORSION MODULES AND RELATIVE PURE-INJECTIVITY
}

\author{
LIXIN MAO and NANQING DING ${ }^{\Xi}$
}

(Received 29 January 2005; revised 2 June 2005)

Communicated by J. Du

\begin{abstract}
Let $R$ be a ring. A right $R$-module $C$ is called a cotorsion module if $\operatorname{Ext}_{R}^{1}(F, C)=0$ for any flat right $R$-module $F$. In this paper, we first characterize those rings satisfying the condition that every cotorsion right (left) module is injective with respect to a certain class of right (left) ideals. Then we study relative pure-injective modules and their relations with cotorsion modules.
\end{abstract}

2000 Mathematics subject classification: primary 16D50; secondary 16E50.

Keywords and phrases: cotorsion module, relative pure-injective module, $P S$ ring, universally mininjective ring, pure- $V$-ring.

\section{Introduction}

Throughout this paper, all rings are associative with identity and all modules are unitary.

Let $R$ be a ring. A right $R$-module $C$ is called a cotorsion module [9] if $\operatorname{Ext}_{R}^{1}(F, C)=0$ for any flat right $R$-module $F$. The ring $R$ is called right cotorsion if $R_{R}$ is cotorsion [2]. The class of cotorsion modules contains all pure-injective (and hence all injective) modules, and is closed under finite direct sums and direct summands.

Let $\mathscr{C}$ be a class of right $R$-modules and $M$ a right $R$-module. Following [10], a $\mathscr{C}$ precover of $M$ is a homomorphism $\phi: F \rightarrow M$ with $F \in \mathscr{C}$ such that $\operatorname{Hom}\left(F^{\prime}, F\right) \rightarrow$ $\operatorname{Hom}\left(F^{\prime}, M\right)$ is surjective for all $F^{\prime} \in \mathscr{C}$. The $\mathscr{C}$-precover $\phi$ is said to be a $\mathscr{C}$-cover if any endomorphism $h: F \rightarrow F$, such that $\phi h=\phi$, is an isomorphism. For $\mathscr{C}$ some familiar class of modules, say the class of flat modules, $\mathscr{C}$-covers will simply be called flat covers. $\mathscr{C}$-envelopes of $M$ can be defined dually. The existence of

(c) 2006 Australian Mathematical Society $1446-7887 / 06 \$ A 2.00+0.00$ 
a flat cover and a cotorsion envelope for any module over any associative ring has been recently proven [6]. An important feature of flat covers (respectively cotorsion envelopes) is that their kernels (respectively cokernels) are cotorsion (respectively flat) by Wakamatsu's Lemmas [25, Section 2.1].

In what follows, we write $M_{R}$ to indicate a right $R$-module. $M^{(l)}$ denotes the direct sum of copies of a module $M$ indexed by a set $I$. As usual, $J(M), Z(M)$ and $\operatorname{Soc}(M)$ stand for the Jacobson radical, the singular submodule and the socle of $M$, respectively. For a subset $X$ of $R$, the left (right) annihilator of $X$ in $R$ is denoted by $l(X)$ (respectively $r(X)$ ). If $X=\{a\}$, we usually abbreviate it to $l(a)$ (or $r(a)$ ). We use $K \leq_{e} N$ and $K \leq^{\oplus} N$ to mean that $K$ is an essential submodule and a direct summand of $N$ respectively. For a right $R$-module $M, \varepsilon_{M}: F(M) \rightarrow M$ and $\sigma_{M}: M \rightarrow C(M)$ denote a flat cover and a cotorsion envelope of $M$ respectively. We frequently identify $M$ with its image in $C(M)$ and think of $M$ as a submodule of $C(M)$. For other definitions and notations, we refer the reader to $[1,10,21,25]$ as background references.

In Section 2, we study rings such that every cotorsion right (left) module is injective with respect to a certain class of right (left) ideals. For example, we show that $R$ is a von Neumann regular ring if and only if every cotorsion right $R$-module is $P$-injective if and only if every non-zero right $R$-module contains a non-zero flat submodule; $R$ is a left $P S$ ring if and only if every cotorsion right $R$-module is $\operatorname{Soc}\left({ }_{R} R\right)$-injective if and only if every left $R$-module $M$ has a monic $\mathscr{M} \mathscr{I}$-cover, where $\mathscr{M} \mathscr{I}$ denotes the class of all mininjective left $R$-modules; $R$ is a left universally mininjective ring if and only if every cotorsion left $R$-module is $\operatorname{Soc}\left({ }_{R} R\right)$-injective if and only if every cotorsion left $R$-module is mininjective if and only if every quotient of any flat cotorsion left $R$-module is $\operatorname{Soc}\left({ }_{R} R\right)$-injective.

In Section 3, we study relative pure-injective modules and their relations with cotorsion modules. Let $M$ and $N$ be right $R$-modules. Recall that $N$ is called $M$ pure-injective if every homomorphism from a pure submodule of $M$ to $N$ can be extended to a homomorphism from $M$ to $N . M$ is said to be quasi-pure-injective if $M$ is $M$-pure-injective. Some useful properties are presented. For instance, let $M_{R}$ be a right $R$-module with endomorphism ring $S$. It is shown that, if $M_{R}$ is quasi-pureinjective, then $S_{S}$ is a quasi-pure-injective right $S$-module; if $M_{R}$ is $M_{R}^{(I)}$-pure-injective for any index set $I$, then $S$ is a right cotorsion ring. We also prove that for a right cotorsion ring $R$, the class of $R$-pure-injective right $R$-modules is closed under direct sums if and only if $R$ is a semiperfect ring; a ring $R$ is right perfect if and only if every right $R$-module has a cotorsion (pre)cover. As a byproduct, we find that every quotient module of any cotorsion (or injective) right $R$-module is cotorsion if and only if every pure submodule of any projective right $R$-module is projective if and only if all flat right $R$-modules are of projective dimension at most 1 . This removes the unnecessary hypothesis that $R$ is a commutative domain from [15, Theorem 3.2]. 
Section 4 is devoted to a new generalization of $V$-rings. A ring $R$ is called a right pure $V$-ring if every simple right $R$-module is $R$-pure-injective. This new class of rings contains right $V$-rings, right perfect rings, commutative rings and semilocal rings. Let $I$ be a right ideal of a ring $R$. Following [17], $I^{*}$ stands for the intersection of all maximal right ideals of $R$ containing $I$. It is shown that $R$ is a right pure-V-ring if and only if $K^{*} \neq P^{*}$ for any maximal submodule $K$ of a pure right ideal $P$ of $R$.

\section{Some properties of cotorsion modules}

Let $\mathscr{A}$ be a nonempty collection of right ideals of a ring $R$. Following [23], a right $R$-module $X$ is said to be $\mathscr{A}$-injective provided that each $R$-homomorphism $f: A \rightarrow X$ with $A \in \mathscr{A}$ extends to $R$, or equivalently, $\operatorname{Ext}_{R}^{1}(R / A, X)=0$ for any $A \in \mathscr{A}$. In particular, $X$ is called $P$-injective (respectively, mininjective) [19] if $X$ is $\mathscr{A}$-injective with $\mathscr{A}=$ all principal right ideals of $R$ \} (respectively, \{all simple right ideals of $R\}$ ), and $X$ is said to be $\operatorname{Soc}\left(R_{R}\right)$-injective (respectively, $\operatorname{Soc}\left({ }_{R} R\right)$-injective) if $\mathscr{A}=\left\{\operatorname{Soc}\left(R_{R}\right)\right\}$ (respectively, $\mathscr{A}=\left\{\operatorname{Soc}\left({ }_{R} R\right)\right\}$ ).

LEMMA 2.1 ([16, Proposition 2.10]). Let $R$ be a ring and $\mathscr{A}$ a nonempty collection of right ideals of $R$. Then the following are equivalent:

(1) Every cotorsion right $R$-module is $\mathscr{A}$-injective.

(2) Every pure-injective right $R$-module is $\mathscr{A}$-injective.

(3) $R / A$ is a flat right $R$-module for any $A \in \mathscr{A}$.

PROPOSITION 2.2. Let $\mathscr{A}$ be a nonempty collection of right ideals of a ring $R$ such that $A$ is projective for any $A \in \mathscr{A}$. If $R_{R}$ is $\mathscr{A}$-injective, then every cotorsion right $R$-module is $\mathscr{A}$-injective. The converse holds if $R$ is right cotorsion.

PROOF. It is enough to show that every right ideal $A$ in $\mathscr{A}$ is a pure submodule of $R$ by Lemma 2.1. Consider the equations

$$
a_{j}=\sum_{i} b_{i} s_{i j}
$$

with $a_{j} \in A, b_{i} \in R, s_{i j} \in R$ for all $1 \leq j \leq n, 1 \leq i \leq m$. Since $A$ is projective, by the Dual Basis Lemma (see [14, 2B2.9, page 23]), there exist a family of elements $\left\{c_{k}: k \in I\right\} \subseteq A$ and linear functionals $\left\{f_{k}: k \in I\right\} \subseteq \operatorname{Hom}_{R}(A, R)$ such that for any $c \in A, f_{k}(c)=0$ for almost all $k$, and $c=\sum_{k} c_{k} f_{k}(c)$. Since $R_{R}$ is $\mathscr{A}$-injective, there are $g_{k} \in \operatorname{Hom}_{R}(R, R)$ such that

$$
f_{k}\left(a_{j}\right)=g_{k}\left(a_{j}\right)=g_{k}\left(\sum_{i} b_{i} s_{i j}\right)=\sum_{i} g_{k}\left(b_{i}\right) s_{i j} .
$$


Therefore

$$
a_{j}=\sum_{k} c_{k} f_{k}\left(a_{j}\right)=\sum_{k} c_{k} \sum_{i} g_{k}\left(b_{i}\right) s_{i j}=\sum_{i}\left(\sum_{k} c_{k} g_{k}\left(b_{i}\right)\right) s_{i j} .
$$

It follows that $A$ is a pure submodule of $R$ by [14, Theorem 4.89].

The last statement is clear.

A ring $R$ is called right $P P$ if every principal right ideal is projective. $R$ is said to be a right $S F$ ring (respectively, right $V$-ring) if every simple right $R$-module is flat (respectively, injective). $R$ is called right semi-artinian if every nonzero right $R$-module contains a nonzero simple submodule.

In [8], it was proved that a ring $R$ is a right semi-artinian right $V$-ring if and only if every nonzero right $R$-module contains a nonzero injective submodule. Motivated by this, we have the following result.

THEOREM 2.3. Let $R$ be a ring. Then the following are equivalent:

(1) $R$ is a von Neumann regular ring.

(2) Every cotorsion right $R$-module is flat.

(3) Every cotorsion right $R$-module is injective.

(4) Every cotorsion right $R$-module is $P$-injective.

(5) $R$ is a right $P P$ right $P$-injective ring.

(6) Every non-zero right $R$-module contains a non-zero flat submodule.

In particular, if $R$ is a right semi-artinian right $S F$ ring, then the above conditions hold.

PROOF. (1) if and only if (3) holds by Lemma 2.1. (1) imples (2), (1) imples (5) and (1) imples (6) are clear.

(5) imples (4) follows from Proposition 2.2.

(2) imples (1). Let $M$ be any right $R$-module. Then there is an exact sequence

$$
0 \longrightarrow M \stackrel{\sigma_{M}}{\longrightarrow} C(M) \longrightarrow L \longrightarrow 0,
$$

where $L$ is flat. Thus $M$ is flat by (2), and (1) follows.

(4) imples (1). Note that $R / A$ is flat for any principal right ideal $A$ by (4) and Lemma 2.1. Thus $R / A$ is projective since $R / A$ is finitely presented. It follows that $A$ is a direct summand of $R$, which implies that $R$ is von Neumann regular.

(6) imples (3). Assume that $0 \rightarrow A \rightarrow B \rightarrow C \rightarrow 0$ is any exact sequence. To simplify the notation, we think of $A$ as a submodule of $B$. Let $M$ be a cotorsion right $R$-module and $f: A \rightarrow M$ be any homomorphism. By a simple application of Zorn's Lemma, we can find some $g: D \rightarrow M$ where $A \subseteq D \subseteq B$, and $\left.g\right|_{A}=f$, such that 
$g$ cannot be extended to any submodule of $B$ properly containing $D$. We claim that $D=B$. Indeed, if $D \neq B$, then $B / D \neq 0$. By (6), there exists a non-zero submodule $N / D$ of $B / D$ such that $N / D$ is flat. Since $M$ is cotorsion, there exists $h: N \rightarrow M$ such that $\left.h\right|_{D}=g$. It is obvious that $h$ extends $g$; this yields the desired contradiction, and so $M$ is injective.

Finally, suppose that $R$ is a right semi-artinian right $S F$ ring. Then every cotorsion right $R$-module is $\mathscr{A}$-injective by Lemma 2.1 , where $\mathscr{A}=$ \{all maximal right ideals of $R$ \}. On the other hand, every $\mathscr{A}$-injective right $R$-module is injective by [23, Lemma 4]. It follows that every cotorsion right $R$-module is injective, as desired.

The equivalences of (1) through (3) in Theorem 2.3 have been shown by Xu (see [25, Theorem 3.3.2]) in a different way.

The following easy observation is given for completeness.

PROPOSITION 2.4. Let $R$ be a ring. Then the following are equivalent:

(1) $R$ is a semisimple Artinian ring.

(2) Every cotorsion right $R$-module is projective.

(3) Every non-zero right $R$-module contains a non-zero projective submodule.

ProOf. (1) implies (2) and (1) implies (3) are clear.

(2) implies (1). $R$ is quasi-Frobenius, since every injective right $R$-module is projective, and $R$ is von Neumann regular by Theorem 2.3 , since every cotorsion right $R$-module is flat. So (1) follows.

(3) implies (1). By the proof of (6) implies (3) in Theorem 2.3, every right $R$-module is injective. Thus $R$ is semisimple Artinian.

A ring $R$ is called left $P S$ [18] if every simple left ideal is projective. It is obvious that $R$ is a left $P S$ ring if and only if $\operatorname{Soc}\left({ }_{R} R\right)$ is projective.

THEOREM 2.5. Let $R$ be a ring. Then the following are equivalent:

(1) $R$ is a left $P S$ ring.

(2) Every cotorsion right $R$-module is $\operatorname{Soc}\left({ }_{R} R\right)$-injective.

(3) Every quotient of any (min)injective left $R$-module is mininjective.

(4) Every quotient of any $\left(\operatorname{Soc}\left({ }_{R} R\right)\right.$-)injective left $R$-module is $\operatorname{Soc}\left({ }_{R} R\right)$-injective.

(5) Every left $R$-module $M$ has a monic $\mathscr{M} \mathscr{I}$-cover, where $\mathscr{M} \mathscr{I}$ denotes the class of all mininjective left $R$-modules.

(6) $R / \operatorname{Soc}\left({ }_{R} R\right)$ is a flat right $R$-module.

(7) $\left(\operatorname{Soc}\left({ }_{R} R\right)\right)^{2}=\operatorname{Soc}\left({ }_{R} R\right)$.

Proof. (2) if and only if (6) if and only if (7) follow from Lemma 2.1 and [5, Proposition 1.4]. 
(3) implies (1). Let $N$ be a submodule of an injective left $R$-module $E$ and $\pi: E \rightarrow E / N$ the canonical map. Suppose that $K$ is a simple left ideal of $R$, and $f: K \rightarrow E / N$ is any homomorphism. Since $E / N$ is mininjective by (3), there exists $g: R \rightarrow E / N$ such that $f=g \iota$ where $\iota: K \rightarrow R$ is the inclusion. It follows that there exists $h: R \rightarrow E$ such that $g=\pi h$, since $R$ is projective. Hence $f=(\pi h) \iota=\pi(h \iota)$ and $K$ is projective by [21, Lemma 4.22].

(1) implies (3). Let $X$ be any mininjective left $R$-module and $N$ any submodule of $X$. We show that $X / N$ is mininjective. To this end, let $K$ be a simple left ideal of $R, i: K \rightarrow R$ the inclusion and $\pi: X \rightarrow X / N$ the canonical map. For any $f: K \rightarrow X / N$, there exists $g: K \rightarrow X$ such that $\pi g=f$, since $K$ is projective by (1). Hence there exists $h: R \rightarrow X$ such that $h i=g$, since $X$ is mininjective. It follows that $(\pi h) i=f$, and (3) holds.

The proof of (1) if and only if (4) is similar to that of (1) if and only if (3).

(1) implies (7). It is clear that $\left(\operatorname{Soc}\left({ }_{R} R\right)\right)^{2} \subseteq \operatorname{Soc}\left({ }_{R} R\right)$. We claim that $\operatorname{Soc}\left({ }_{R} R\right) I \neq 0$ for any simple left ideal $I$. If not, then there exists a simple left ideal $R a$ such that $\operatorname{Soc}\left({ }_{R} R\right) R a=0$. Since $R$ is a left $P S$ ring, we have $R=l_{R}(a) \oplus K$ with $K$ a left ideal of $R$, and so $R a=K a$. On the other hand, $K \cong R / l_{R}(a)$ is simple. Thus $K a=0$, and hence $R a=0$, a contradiction. Therefore, $I=\operatorname{Soc}\left({ }_{R} R\right) I$ for any simple left ideal $I$. It follows that $\operatorname{Soc}\left({ }_{R} R\right) \subseteq\left(\operatorname{Soc}\left({ }_{R} R\right)\right)^{2}$, and hence $\left(\operatorname{Soc}\left({ }_{R} R\right)\right)^{2}=\operatorname{Soc}\left({ }_{R} R\right)$.

(7) implies (1) follows from [5, Proposition 1.10 (b)].

(3) implies (5). Let $M$ be any left $R$-module. Write $F=\sum\{N \leq M: N \in \mathscr{M} \mathscr{I}\}$ and $G=\oplus\{N \leq M: N \in \mathscr{M} \mathscr{I}\}$. Then there exists an exact sequence $0 \rightarrow K \rightarrow$ $G \rightarrow F \rightarrow 0$. Note that $G \in \mathscr{M} \mathscr{I}$, so $F \in \mathscr{M} \mathscr{I}$ by (3). Next we prove that the inclusion $i: F \rightarrow M$ is an $\mathscr{M} \mathscr{I}$-cover of $M$. Let $\psi: F^{\prime} \rightarrow M$, with $F^{\prime} \in \mathscr{M} \mathscr{I}$, be an arbitrary left $R$-homomorphism. Note that $\psi\left(F^{\prime}\right) \leq F$ by (3). Define $\zeta: F^{\prime} \rightarrow F$ via $\zeta(x)=\psi(x)$ for $x \in F^{\prime}$. Then $i \zeta=\psi$, and so $i: F \rightarrow M$ is an $\mathscr{M} \mathscr{I}$-precover of $M$. In addition, it is clear that the identity map $l_{F}$ of $F$ is the only homomorphism $g: F \rightarrow F$ such that $i g=i$, and hence (5) follows.

(5) implies (3). Let $M$ be any mininjective left $R$-module and $N$ any submodule of $M$. We show that $M / N$ is mininjective. Indeed, there exists an exact sequence $0 \rightarrow N \rightarrow E \rightarrow L \rightarrow 0$ with $E$ injective. Since $L$ has a monic $\mathscr{M} \mathscr{I}$-cover $\phi: F \rightarrow L$ by (5), there exists $\alpha: E \rightarrow F$ such that the following exact diagram is commutative:

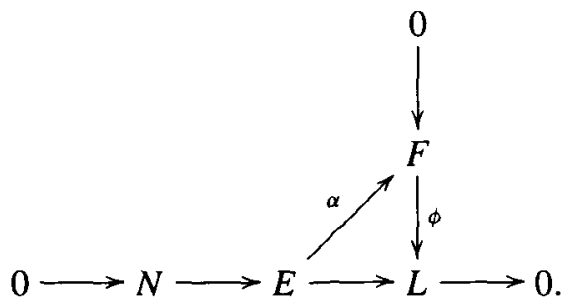


Thus $\phi$ is epic, and hence it is an isomorphism. Therefore $L$ is mininjective. For any $K=R / A$ with $A$ a simple left ideal, we have

$$
0=\operatorname{Ext}_{R}^{1}(K, L) \rightarrow \operatorname{Ext}_{R}^{2}(K, N) \rightarrow \operatorname{Ext}_{R}^{2}(K, E)=0 .
$$

Therefore, $\operatorname{Ext}_{R}^{2}(K, N)=0$. On the other hand, the short exact sequence $0 \rightarrow N \rightarrow$ $M \rightarrow M / N \rightarrow 0$ induces the exactness of the sequence

$$
0=\operatorname{Ext}_{R}^{1}(K, M) \rightarrow \operatorname{Ext}_{R}^{1}(K, M / N) \rightarrow \operatorname{Ext}_{R}^{2}(K, N)=0 .
$$

Therefore, $\operatorname{Ext}_{R}^{1}(K, M / N)=0$, as desired.

Following [19], a ring $R$ is called left universally mininjective if every left $R$ module is mininjective. Recall that a $\mathscr{C}$-envelope $\alpha: M \rightarrow L$ has the unique mapping property [7] if for any homomorphism $f: M \rightarrow N$ with $N \in \mathscr{C}$, there exists a unique $g: L \rightarrow N$ such that $g \alpha=f$.

THEOREM 2.6. Let $R$ be a ring. Then the following are equivalent:

(1) $R$ is a left universally mininjective ring.

(2) Every simple left ideal is generated by an idempotent.

(3) Every cotorsion left $R$-module is $\operatorname{Soc}\left({ }_{R} R\right)$-injective.

(4) Every cotorsion left $R$-module is mininjective.

(5) Every quotient of any cotorsion left $R$-module is $\operatorname{Soc}\left({ }_{R} R\right)$-injective.

(6) Every quotient of any flat cotorsion left $R$-module is $\operatorname{Soc}\left({ }_{R} R\right)$-injective.

(7) Every cotorsion left $R$-module has an $\mathscr{M} \mathscr{I}$-envelope with the unique mapping property.

(8) $R / \operatorname{Soc}\left({ }_{R} R\right)$ is a flat left $R$-module.

(9) $R$ is a left $P S$ left mininjective ring.

Moreover, if $R$ is a left cotorsion ring with $\operatorname{Soc}\left({ }_{R} R\right) \leq_{e R} R$, then the above conditions are also equivalent to:

(10) $R$ is a left $P S$ left $\operatorname{Soc}\left({ }_{R} R\right)$-injective ring.

(11) $R$ is a left mininjective left nonsingular ring.

(12) $R$ is a left mininjective ring with $J(R)=0$.

(13) $R$ is a von Neumann regular ring.

Proof. (1) implies (7), (2) implies (9) and (5) implies (6) are trivial.

(2) if and only if (8) if and only if (3) hold by [5, Proposition 2.1] and Lemma 2.1.

(9) implies (4) follows from Proposition 2.2.

(1) implies (2). Let $S$ be a simple left ideal. Then $S$ is mininjective, and so $S$ is a direct summand of $R$. Thus (2) holds. 
(7) implies (4). Let $M$ be any cotorsion left $R$-module. There is the following exact commutative diagram

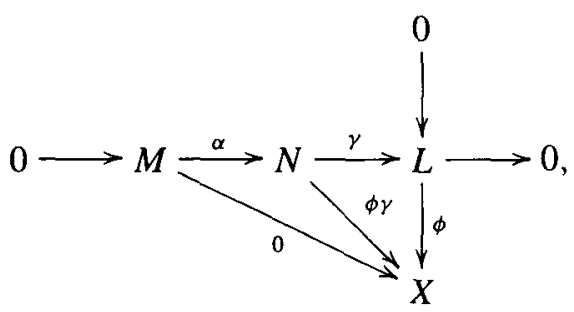

where $\alpha$ and $\phi$ are $\mathscr{M} \mathscr{I}$-envelopes with the unique mapping property. Note that $\phi \gamma \alpha=0=0 \alpha$, so $\phi \gamma=0$ by (7). Therefore $L=\operatorname{im}(\gamma) \subseteq \operatorname{ker}(\phi)=0$, and so $M$ is mininjective. Hence (4) follows.

(4) implies (1). Note that $R / A$ is flat for any simple left ideal $A$ by (4) and Lemma 2.1. Thus $R / A$ is projective since $R / A$ is finitely presented. It follows that $A$ is a direct summand of $R$, which implies that $R$ is left universally mininjective.

(3) implies (5). Note that (3) if and only if (9) holds by the preceding proof. Let $M$ be any cotorsion left $R$-module and $N$ any submodule of $M$. We show that $M / N$ is $\operatorname{Soc}\left({ }_{R} R\right)$-injective. To this end, let $\pi: M \rightarrow M / N$ be the canonical map and $i: \operatorname{Soc}\left({ }_{R} R\right) \rightarrow{ }_{R} R$ the inclusion. For any $f \in \operatorname{Hom}_{R}\left(\operatorname{Soc}\left({ }_{R} R\right), M / N\right)$, there exists $g: \operatorname{Soc}\left({ }_{R} R\right) \rightarrow M$ such that $\pi g=f$, since $\operatorname{Soc}\left({ }_{R} R\right)$ is projective by $(9)$. Hence there exists $h:{ }_{R} R \rightarrow M$ such that $h i=g$, since $M$ is $\operatorname{Soc}\left({ }_{R} R\right)$-injective by (3). It follows that $(\pi h) i=f$, and so $(5)$ holds.

(6) implies (3). Let $M$ be any cotorsion left $R$-module. Then $M$ has a flat cover $\varepsilon_{M}: F(M) \rightarrow M$. Since $\operatorname{ker}\left(\varepsilon_{M}\right)$ is cotorsion, by Wakamatsu's Lemma, $F(M)$ is both flat and cotorsion. So $M$ is $\operatorname{Soc}\left({ }_{R} R\right)$-injective by (6).

(3) if and only if (10) holds by Proposition 2.2.

(9) implies (11). Note that $Z_{l}\left(\operatorname{Soc}\left({ }_{R} R\right)\right)=Z_{l}(R) \cap \operatorname{Soc}\left({ }_{R} R\right)$ and $Z_{l}\left(\operatorname{Soc}\left({ }_{R} R\right)\right)=0$ by [14, Exercise $12 \mathrm{~A}(\mathrm{c})$, page 269] and (9), so $Z_{l}(R) \cap \operatorname{Soc}\left({ }_{R} R\right)=0$, and hence $Z_{l}(R)=0$ since $\operatorname{Soc}\left({ }_{R} R\right) \leq_{e} R$.

(11) implies (9) follows from [18, Example 2.5 (3)].

(11) if and only if (12). For a left cotorsion ring with $\operatorname{Soc}\left({ }_{R} R\right) \leq_{e} R$, we can prove a more general result: $Z_{l}(R)=J(R)=r_{R}\left(\operatorname{Soc}\left({ }_{R} R\right)\right)$. In fact, since $R$ is left mininjective ring, $\operatorname{Soc}\left({ }_{R} R\right) \subseteq \operatorname{Soc}\left(R_{R}\right)$ by [19, Theorem 2.21(c)]. It follows that $J(R) \leq r_{R}\left(\operatorname{Soc}\left(R_{R}\right)\right) \leq r_{R}\left(\operatorname{Soc}\left({ }_{R} R\right)\right)$. In addition, $Z_{l}(R) \leq J(R)$ by [19, Theorem B.58] or the remark just before [2, Theorem 6], and $r_{R}\left(\operatorname{Soc}\left({ }_{R} R\right)\right) \leq Z_{l}(R)$ by $\operatorname{Soc}\left({ }_{R} R\right) \leq_{e} R$. Thus (11) if and only if (12) follows.

(13) implies (12) is obvious.

(12) implies (13). Note that $R / J(R)$ is von Neumann regular by [2, Theorem 6]. The proof is complete. 
REMARK 2.7. It is obvious that a left universally mininjective ring is left $P S$ by Theorem 2.6. However, the converse is not true as shown by the following example.

Let

$$
R=\left(\begin{array}{ll}
\mathbb{Z}_{2} & 0 \\
\mathbb{Z}_{2} & \mathbb{Z}_{2}
\end{array}\right)=\left\{\left(\begin{array}{ll}
a & 0 \\
b & c
\end{array}\right): a, b, c \in \mathbb{Z}_{2}\right\}
$$

and $x=\left(\begin{array}{ll}0 & 0 \\ 1 & 0\end{array}\right) \in R$. It is easy to see that $R x$ is a simple left ideal, and $R x$ can not be generated by an idempotent, so $R x$ is not mininjective. However, $R$ is a left $P P$, and hence a left $P S$ ring. In fact, it is easily checked that every element of $R$ is either nilpotent or idempotent or invertible. Note that $x=\left(\begin{array}{ll}0 & 0 \\ 1 & 0\end{array}\right)$ is the only non-zero nilpotent element and $l(x)=R\left(\begin{array}{ll}1 & 0 \\ 0 & 0\end{array}\right)$ is a summand of $R$, and so $R x$ is projective, as required.

COROLLARY 2.8. Let $R$ be a commutative ring, then the following are equivalent:

(1) $R$ is a PS ring.

(2) $R$ is a universally mininjective ring.

(3) Every cotorsion $R$-module is $\operatorname{Soc}(R)$-injective.

(4) Every cotorsion $R$-module is mininjective.

PROOF. The result follows from Theorem 2.5 and Theorem 2.6.

\section{Relative pure-injective modules}

In this section, we investigate the pure injectivity relative to a module and discuss its relationship with cotorsion modules. We first recall the following definition (see, for example, [24]).

DEFINITION 3.1. Let $M$ and $N$ be right $R$-modules. $N$ is called $M$-pure-injective if every homomorphism $f: K \rightarrow N$, where $K$ is a pure submodule of $M$, can be extended to a homomorphism $g: M \rightarrow N$.

$M$ is called quasi-pure-injective if $M$ is $M$-pure-injective.

Clearly, if $N$ is $M$-injective, then $N$ is $M$-pure-injective. The next proposition is easy to verify.

Proposition 3.2. Let $M$ and $N$ be right $R$-modules. Then

(1) $N$ is pure-injective if and only if $N$ is $M$-pure-injective for all right $R$-modules $M$.

(2) $N$ is cotorsion if and only if $N$ is $M$-pure-injective for all free (respectively, projective, flat) right $R$-modules $M$. 
It is obvious that the ring $\mathbb{Z}$ of integers (more generally, any domain) has no nontrivial pure ideal, so every $\mathbb{Z}$-module is $\mathbb{Z}$-pure-injective. However, $\mathbb{Z}$ is not cotorsion.

PROPOSITION 3.3. For a right $R$-module $M$, the following are equivalent:

(1) Every pure submodule of $M$ is a direct summand of $M$.

(2) Every right $R$-module is $M$-pure-injective.

(3) Every pure submodule of $M$ is $M$-pure-injective.

In particular, if $M$ is a finitely generated projective right $R$-module, then the above conditions are equivalent to:

(4) Every pure submodule of $M$ is finitely generated.

(5) Every flat quotient module of $M$ is projective.

PROOF. The proof is straightforward and hence omitted.

Some general properties of this kind of relative pure-injectivity follow below.

PROPOSITION 3.4. Let $M$ and $N$ be right $R$-modules. If $N$ is $M$-pure-injective, then for every pure submodule $K$ of $M, N$ is $K$-pure-injective and $M / K$-pure-injective.

Proof. Every pure submodule of $K$ is also a pure submodule of $M$ since $K$ is a pure submodule of $M$. Therefore it is clear that $N$ is $K$-pure-injective.

Now let us prove that $N$ is $M / K$-pure-injective. Let $L / K$ be any pure submodule of $M / K$ and $f: L / K \rightarrow N$ any homomorphism. By [14, Exercise 30, page 162], $L$ is a pure submodule of $M$. Let $\pi_{1}: M \rightarrow M / K$ and $\pi_{2}: L \rightarrow L / K$ be the canonical maps. Since $N$ is $M$-pure-injective, there is a homomorphism $g: M \rightarrow N$ that extends $f \pi_{2}$. Note that $K \leq \operatorname{ker}(g)$, hence there exists $h: M / K \rightarrow N$ such that $h \pi_{1}=g$. For any $l \in L, h(l+K)=h \pi_{1}(l)=g(l)=f \pi_{2}(l)=f(l+K)$. Thus $h$ extends $f$, and so $N$ is $M / K$-pure-injective.

The next lemma is easy to verify.

LEMMA 3.5. Let $M$ be a right $R$-module and $\left\{N_{i}: i \in I\right\}$ a family of right $R$ modules. Then $\prod_{i \in I} N_{i}$ is $M$-pure-injective if and only if $N_{i}$ is $M$-pure-injective for every $i \in I$.

In particular, a direct summand of an $M$-pure-injective right $R$-module is $M$-pureinjective.

REMARK 3.6. In general, the class of $M$-pure-injective modules is not closed under direct sums. For example, let $R$ be a von Neumann regular ring, but not right Noetherian. Then the class of $R$-pure-injective right modules is not closed under direct sums. 
It is well-known that $M_{1} \oplus M_{2}$ is quasi-injective if and only if $M_{i}$ is $M_{j}$-injective for all $i, j=1,2$.

PROPOSITION 3.7. Let $M_{1}$ and $M_{2}$ be right $R$-modules. If $M_{1} \oplus M_{2}$ is quasi-pureinjective, then $M_{i}$ is $M_{j}$-pure-injective for all $i, j=1,2$.

PrOOF. This follows from Proposition 3.4 and Lemma 3.5.

Proposition 3.8. Suppose that $N$ is $M$-pure-injective. If $K$ is a pure submodule of $M$ with $K \cong L$ and $L \leq^{\oplus} N$, then $K \leq^{\oplus} M$.

Proof. Let $i: K \rightarrow M$ and $\iota: L \rightarrow N$ be the inclusions, $\pi: N \rightarrow L$ the canonical projection and $f: K \rightarrow L$ the isomorphism. Since $N$ is $M$-pure-injective, there exists $g: M \rightarrow N$ such that $g i=\imath f$. Let $\alpha=f^{-1} \pi g: M \rightarrow K$. For any $k \in K, \alpha i(k)=f^{-1} \pi g i(k)=f^{-1} f(k)=k$. So $\alpha i=1_{K}$, which implies that $K \leq^{\oplus} M$.

COROLLARY 3.9. If $M$ is a quasi-pure-injective right $R$-module, then $M$ is pure- $C 2$, that is, assume that $K$ is a pure submodule of $M$ with $K \cong L$ and $L \leq^{\oplus} M$, then $K \leq{ }^{\oplus} M$.

PROPOSITION 3.10. Let $M$ be a flat cotorsion right $R$-module. Then

(1) $M$ is pure- $C 2$.

(2) (pure-C 3) If $K$ and $L$ are submodules of $M$ with $K \cap L=0, K \leq^{\oplus} M$ and $L \leq^{\oplus} M$, then $K \oplus L$ is a pure submodule of $M$ if and only if $K \oplus L \leq^{\oplus} M$.

ProOF. (1) Since $M$ is flat and cotorsion, then $M$ is quasi-pure-injective by Proposition 3.2. So (1) follows from Corollary 3.9.

(2) Let $K=e M, e^{2}=e \in \operatorname{End}\left(M_{R}\right)$, so that $K \oplus L=e M \oplus(1-e) L$. Thus $(1-e) L \cong L \leq^{\oplus} M$. If $K \oplus L$ is a pure submodule of $M$, then $(1-e) L$ is also a pure submodule of $M$. By $(1),(1-e) L \leq^{\oplus} M$, and so there exists $f^{2}=f \in \operatorname{End}\left(M_{R}\right)$ such that $(1-e) L=f M$. Thus $e f=0$, and hence $h=e+f-f e$ is an idempotent and $K \oplus L=h M \leq \leq^{\oplus} M$. The converse is clear.

COROLLARY 3.11. If $R$ is a right cotorsion ring, then $R_{R}$ is pure-C 2 and pure-C3.

PROPOSITION 3.12. Let $M$ be a flat right $R$-module and $N$ a right $R$-module. If $\alpha(M) \subseteq N$ for all $\alpha: C(M) \rightarrow C(N)$, then $N$ is $M$-pure-injective.

Proof. Let $K$ be a pure submodule of $M$ and $i: K \rightarrow M$ the inclusion. Note that $M$ is a pure submodule of $C(M)$, so $K$ is also a pure submodule of $C(M)$. Since 
$M$ is flat, so is $C(M)$. Consequently, for any homomorphism $f: K \rightarrow N$, there exists $g: C(M) \rightarrow C(N)$ such that $g \sigma_{M} i=\sigma_{N} f$. By hypothesis, $g(M) \subseteq N$. So $\left.g\right|_{M}: M \rightarrow N$ extends $f$, as desired.

COROLlaRY 3.13. Let $M$ be a flat right $R$-module. If $M$ is a fully invariant submodule of $C(M)$, then $M$ is quasi-pure-injective.

REMARK 3.14. From the proof of Proposition 3.12, Corollary 3.13 can be extended to a more general result, that is, a fully invariant pure submodule of a quasi-pureinjective right $R$-module is quasi-pure-injective. On the other hand, it is well known that a module $M$ is quasi-injective if and only if $M$ is a fully invariant submodule of its injective envelope. However, we do not know whether the converse of Corollary 3.13 is true.

PROPOSITION 3.15. Let ${ }_{S} F_{R}$ be a bimodule and $M_{R}$ a right $R$-module.

(1) If $M_{R}$ is $F_{R}$-pure-injective, then $\operatorname{Hom}_{R}\left({ }_{s} F_{R}, M_{R}\right)$ is an $S$-pure-injective right $S$-module.

(2) If $M_{R}$ is $F_{R}^{(I)}$-pure-injective for any index set $I$, then $\operatorname{Hom}_{R}\left({ }_{s} F_{R}, M_{R}\right)$ is a cotorsion right $S$-module.

PROOF. (1) Let $K_{S}$ be a pure submodule of $S_{S}$. We can consider the right $R$ module $K \otimes_{S} F$ as a pure submodule in the right $R$-module $S \otimes_{S} F$. Since $M_{R}$ is $F_{R}$-pure-injective and $S \otimes_{S} F \cong F_{R}$, we obtain the exact sequence

$$
\operatorname{Hom}_{R}\left(S \otimes_{S} F_{R}, M_{R}\right) \rightarrow \operatorname{Hom}_{R}\left(K \otimes_{S} F_{R}, M_{R}\right) \rightarrow 0,
$$

which gives rise to the exactness of the sequence

$$
\operatorname{Hom}_{S}\left(S_{S}, \operatorname{Hom}_{R}\left({ }_{S} F_{R}, M_{R}\right)\right) \rightarrow \operatorname{Hom}_{S}\left(K_{S}, \operatorname{Hom}_{R}\left({ }_{s} F_{R}, M_{R}\right)\right) \rightarrow 0 .
$$

Thus $\operatorname{Hom}_{R}\left({ }_{S} F_{R}, M_{R}\right)$ is an $S$-pure-injective right $S$-module.

(2) If $M_{R}$ is $F_{R}^{(I)}$-purc-injective for any index set $I$, then, by the proof of (1), $\operatorname{Hom}_{R}\left({ }_{S} F_{R}, M_{R}\right)$ is an $S^{(I)}$-pure-injective right $S$-module for any index set $I$. So (2) follows from Proposition 3.2.

COROLLARY 3.16. Let $M_{R}$ be a right $R$-module with endomorphism ring $S$.

(1) If $M_{R}$ is quasi-pure-injective, then $S_{S}$ is a quasi-pure-injective right $S$-module.

(2) If $M_{R}$ is $M_{R}^{(I)}$-pure-injective for any index set $I$, then $S$ is a right cotorsion ring. In particular, $S=\operatorname{End}\left(M_{R}\right)$ is a right cotorsion ring for any flat cotorsion right $R$-module $M$. 
Recall that a ring $R$ is said to be $I$-finite [19] if $R$ has no infinite set of nonzero orthogonal idempotents.

LEMMA 3.17. Let $R$ be a ring. If the class of $R$-pure-injective right $R$-modules is closed under direct sums, then

(1) for any ascending chain $I_{1} \subseteq I_{2} \subseteq I_{3} \subseteq \cdots$ of right ideals with $\bigcup_{k=1}^{\infty} I_{k}$ pure in $R$, there is an $n$ with $I_{n+i}=I_{n}, i=1,2, \ldots$;

(2) $R$ is I-finite.

PROOF. (1) Let $I_{1} \subseteq I_{2} \subseteq I_{3} \subseteq \cdots$ be an ascending chain of right ideals with $I=\bigcup_{k=1}^{\infty} I_{k}$ pure in $R$. Define a homomorphism

$$
\begin{aligned}
f: I & \rightarrow \bigoplus_{k=1}^{\infty} C\left(R / I_{k}\right) \\
a & \mapsto\left(a+I_{k}\right)_{k=1}^{\infty}
\end{aligned}
$$

for $a \in I$. By hypothesis, $\oplus_{k=1}^{\infty} C\left(R / I_{k}\right)$ is $R$-pure-injective. So there exists $x \in$ $\oplus_{k=1}^{\infty} C\left(R / I_{k}\right)$ such that $f(a)=x a=\left(a+I_{k}\right)_{k=1}^{\infty}$ for any $a \in I$.

Let $x=\left(x_{1}, x_{2}, \ldots, x_{n}, 0, \ldots\right)$. Then $a+I_{n+k}=0$ for any $a \in I$ and $k \geq 1$. Thus $I=I_{n+1}=I_{n+2}=\cdots$, as desired.

(2) By (1) and [1, Exercise 19.11(1)], $R$ satisfies ACC (ascending chain condition) on pure right ideals. Thus $R$ satisfies ACC on right direct summands, and hence $R$ is $I$-finite by [14, Proposition 6.59].

For any ring $R$, it is easy to see that every cyclic flat right $R$-module is projective if and only if every right $R$-module is $R$-pure-injective (see Proposition 3.3). So we have the following result (see, for example, [20, Lemma 4.5]).

COROLLARY 3.18. If every cyclic flat right $R$-module is projective, then $R$ is $I$-finite. The converse holds when $R$ is a right or left PP ring.

Proof. The result follows from Lemma 3.17, [14, Proposition 6.59 and Theorem 7.55], and [26, Proposition 9].

THEOREM 3.19. If $R$ is a right cotorsion ring, then the following are equivalent:

(1) The class of $R$-pure-injective right $R$-modules is closed under direct sums.

(2) Every right $R$-module is $R$-pure-injective.

(3) $R$ is a semiperfect ring.

(4) $R$ is I-finite. 
PROOF. (2) implies (1) is trivial. (1) implies (4) follows from Lemma 3.17.

(3) implies (2). Since $R$ is semiperfect, every cyclic flat right $R$-module is projective by [14, Exercise 21, page 161] or [20, Example 3.7]. Hence (2) follows.

(4) implies (3). Since $R$ is $I$-finite, $R_{R}=I_{1} \oplus I_{2} \oplus \cdots \oplus I_{n}$ such that $I_{i}$ is indecomposable and $I_{i}=e_{i} R, e_{i}^{2}=e_{i}, i=1, \ldots, n$, by [14, Proposition 6.60]. Let $S_{i}=\operatorname{End}\left(I_{i}\right)$. Then $S_{i}$ is a right cotorsion ring by Corollary 3.16, since each $I_{i}$ is a flat cotorsion right $R$-module, $i=1, \ldots, n$. In addition, 0 and 1 are the only idempotents in $S_{i}$ since $I_{i}$ is indecomposable. It follows that $S_{i}$ is local by [2, Corollary 7]. Each $e_{i} R e_{i} \cong S_{i}, i=1, \ldots, n$. Consequently, $R$ is a semiperfect ring by [1, Theorem 27.6].

Recall that a ring $R$ is a right perfect ring if and only if every right $R$-module is cotorsion, by [25, Proposition 3.3.1]. Thus we have the following result.

THEOREM 3.20. Let $R$ be a ring. Then the following are equivalent:

(1) $R$ is a right perfect ring.

(2) $R$ is a right cotorsion ring with $J(R)$ right $T$-nilpotent, and the class of $R$-pureinjective right $R$-modules is closed under direct sums.

(3) The class of cotorsion right $R$-modules is closed under direct sums.

(4) Every right $R$-module has a cotorsion (pre)cover.

Moreover, if $R$ satisfies $\operatorname{Soc}\left({ }_{R} R\right) \leq_{e} R_{R}$, then the above conditions are also equivalent to:

(5) $R$ is a right cotorsion ring and satisfies ACC for chains of annihilators of the form $r_{R}\left(a_{1}\right) \subseteq r_{R}\left(a_{2} a_{1}\right) \subseteq r_{R}\left(a_{3} a_{2} a_{1}\right) \subseteq \cdots$.

PROOF. (1) if and only if (2) follows from Theorem 3.19.

(1) implies (4) is trivial, (4) implies (3) follows from [22, Proposition 1], and (3) implies (1) holds by [3, Theorem 19].

(1) implies (5) follows from [4, Corollary 25].

(5) implies (1). By [26, Proposition 9], every cyclic flat right $R$-module is projective. Therefore $R$ is a semiperfect ring by Corollary 3.18 and Theorem 3.19. So it is enough to show that $J(R)$ is right T-nilpotent.

Now let $a_{1}, a_{2}, a_{3}, \ldots$ be an infinite sequence in $J(R)$. Then we get a chain

$$
r_{R}\left(a_{1}\right) \subseteq r_{R}\left(a_{2} a_{1}\right) \subseteq r_{R}\left(a_{3} a_{2} a_{1}\right) \subseteq \cdots
$$

Thus there exists $n \in \mathbb{N}$ such that $r_{R}\left(a_{n} a_{n-1} \cdots a_{1}\right)=r_{R}\left(a_{n+1} a_{n} \cdots a_{1}\right)$ by (4), and hence $\left(a_{n} a_{n-1} \cdots a_{1}\right) R \cap r_{R}\left(a_{n+1}\right)=0$. On the other hand, noting that

$$
J(R) \leq l_{R}\left(\operatorname{Soc}{ }_{R} R\right) \leq Z_{r}(R)
$$


by $\operatorname{Soc}\left({ }_{R} R\right) \leq_{e} R_{R}$, we have $a_{n+1} \in Z_{r}(R)$. Thus $r_{R}\left(a_{n+1}\right) \leq_{e} R_{R}$, and so $a_{n} \cdots a_{1}=0$, which implies that $J(R)$ is right T-nilpotent. This completes the proof.

COROLlaRY 3.21. Let $R$ be a commutative ring. Then the following are equivalent:

(1) $R$ is a perfect ring.

(2) $R$ is a cotorsion ring with essential socle and satisfies ACC for chains of annihilators of the form $\operatorname{ann}_{R}\left(a_{1}\right) \subseteq \operatorname{ann}_{R}\left(a_{2} a_{1}\right) \subseteq \operatorname{ann}_{R}\left(a_{3} a_{2} a_{1}\right) \subseteq \cdots$.

PROOF. This follows from [1, Theorem 28.4] and Theorem 3.20.

LEMMA 3.22. Let $M$ be a projective right $R$-module. Then the following are equivalent:

(1) Every quotient module of any injective $R$-module is $M$-pure-injective.

(2) Every quotient module of any $M$-pure-injective right $R$-module is $M$-pureinjective.

(3) Every pure submodule of $M$ is projective.

ProOF. (2) implies (1) is trivial.

The proof of (1) implies (3) implies (2) is similar to that of (1) if and only if (3) in Theorem 2.5.

THEOREM 3.23. Let $R$ be a ring. Then the following are equivalent:

(1) Every quotient module of any injective right $R$-module is cotorsion.

(2) Every quotient module of any cotorsion right $R$-module is cotorsion.

(3) Every pure submodule module of any projective right $R$-module is projective.

(4) All flat right $R$-modules are of projective dimension at most 1 .

(5) $\operatorname{Ext}_{R}^{2}(M, N)=0$ for all flat right $R$-modules $M$ and $N$.

(6) $\operatorname{Ext}_{R}^{j}(M, N)=0$ for all flat right $R$-modules $M, N$ and $j \geq 2$.

PROOF. (1) if and only if (2) if and only if (3) follow from Lemma 3.22.

(4) implies (3). Let $M$ be a projective right $R$-module and $N$ a pure submodule of $M$. Then $0 \rightarrow N \rightarrow M \rightarrow M / N \rightarrow 0$ is exact. Note that $M / N$ is flat, and hence the projective dimension of $M / N$ is less than or equal to 1, by (4). Thus $N$ is projective.

(3) implies (4). Let $M$ be any flat right $R$-module. There exists an exact sequence $0 \rightarrow N \rightarrow P \rightarrow M \rightarrow 0$ with $P$ projective. Note that $N$ is a pure submodule of $P$, so $N$ is projective. It follows that the projective dimension of $M$ is at most 1 .

(4) implies (5) implies (6) are trivial. 
(6) implies (4). Let $M$ be any flat right $R$-module and $N$ any right $R$-module. Then there is an exact sequence

$$
0 \longrightarrow K \longrightarrow F(N) \stackrel{\varepsilon_{N}}{\longrightarrow} N \longrightarrow 0,
$$

with $K$ cotorsion, which induces the exactness of sequence

$$
\operatorname{Ext}_{R}^{2}(M, F(N)) \rightarrow \operatorname{Ext}_{R}^{2}(M, N) \rightarrow \operatorname{Ext}_{R}^{3}(M, K) .
$$

Note that $\operatorname{Ext}_{R}^{2}(M, F(N))=0$, by $(6)$, and $\operatorname{Ext}_{R}^{3}(M, K)=0$, by the proof of [25, Proposition 3.1.2]. So $\operatorname{Ext}_{R}^{2}(M, N)=0$ and (4) follows.

REMARK 3.24. The equivalences of (1) through (4) in the previous theorem have recently been proven for commutative domains ([15, Theorem 3.2]).

\section{A new generalization of $V$-rings}

We start with the following definition.

Definition 4.1. A ring $R$ is called a right pure- $V$-ring if every simple right $R$ module is $R$-pure-injective.

REMARK 4.2. (1) It is obvious that the class of right pure- $V$-rings contains right $V$-rings and right perfect rings. In general, a right pure- $V$-ring need not be a right $V$-ring (for example, $\mathbb{Z}$ ). If $R$ is a von Neumann regular ring, then $R$ is a right $V$-ring if and only if $R$ is a right pure- $V$-ring if and only if every simple right $R$-module is cotorsion.

(2) $[16$, Lemma 2.14] shows that every simple $R$-module over a commutative ring $R$ is cotorsion. So commutative rings are pure- $V$-rings. However, simple $R$-modules over a noncommutative ring $R$ need not be cotorsion. For example, we can choose $R$ to be a von Neumann regular ring, which is not a right $V$-ring (see [11]).

A ring is called semilocal if $R / J(R)$ is a semisimple Artinian ring.

PROPOSITION 4.3. Any semilocal ring $R$ is a left and right pure- $V$-ring.

PROOF. By the Wedderburn-Artin Theorem and [12, Proposition 9.3.4], for any simple right $R$-module $M$ with endomorphism ring $S,{ }_{S} M$ is a finite dimensional vector space. Thus $M$ is $\Sigma$-pure-injective by [13, Lemma 4.3] and therefore cotorsion. So $R$ is a right pure- $V$-ring. Similarly, $R$ is a left pure- $V$-ring. 
Let $I$ be a right ideal of $R$. Following [17], $I^{*}$ stands for the intersection of all maximal right ideals of $R$ containing $I$. Yue Chi Ming proved that $R$ is a right $V$-ring if and only if, for any maximal submodule $K$ of an essential right ideal $P, K^{*} \neq P^{*}$ (see [17, Theorem 3]). Next we give a corresponding characterization of a right pure- $V$-ring.

THEOREM 4.4. Let $R$ be a ring. Then the following are equivalent:

(1) $R$ is a right pure- $V$-ring.

(2) If $K$ is a maximal submodule of a pure right ideal $P$ of $R$, then $K^{*} \neq P^{*}$.

Proof. (1) implies (2). Suppose that there exist a pure right ideal $P$ and a maximal submodule $K$ of $P$ such that $K^{*}=P^{*}$. Then $P / K$ is simple. By (1), there exists $f: R \rightarrow P / K$, which extends the canonical projection $\pi: P \rightarrow P / K$. Let $g=\left.f\right|_{P^{*}}$. Then $K \subseteq \operatorname{ker}(g) \subseteq P^{*}=K^{*}$. Therefore $(\operatorname{ker}(g))^{*}=P^{*}=K^{*}\left(\right.$ for $\left.K^{* *}=K^{*}\right)$. On the other hand, $\operatorname{ker}(f)$ is a maximal right ideal of $R$ with $\operatorname{ker}(f) \cap P^{*}=\operatorname{ker}(g)$. Thus $(\operatorname{ker}(g))^{*} \subseteq \operatorname{ker}(f)$, and hence $P^{*}=(\operatorname{ker}(g))^{*}=\operatorname{ker}(g)$, which implies that $g=0$, and so $P / K=0$, a contradiction.

(2) implies (1). Suppose that $M$ is any simple right $R$-module. Let $I$ be any pure right ideal, and $\alpha: I \rightarrow M$ any homomorphism. We show that $\alpha$ can be extended to $R$.

If $\alpha=0$, this is trivial.

If $\alpha \neq 0$, then $\operatorname{ker}(\alpha)$ is a maximal submodule of $I$, and so $(\operatorname{ker}(\alpha))^{*} \neq I^{*}$ by (2). Thus there exists a maximal right ideal $K$ of $R$ such that $\operatorname{ker}(\alpha) \subseteq K$ and $I \nsubseteq K$. So $R=K+I$. Let $r \in R$. Then there exist $k \in K$ and $t \in I$ such that $r=k+t$. Now we define $\beta: R \rightarrow M$ via $r \mapsto \alpha(t)$. Note that $K \cap I=\operatorname{ker}(\alpha)$. It is easy to verify that $\beta$ is well-defined. Clearly, $\beta$ extends $\alpha$.

COROLLARY 4.5. Let $R$ be a right pure-V-ring and $I^{*} \leq^{\oplus} R_{R}$. Then $I=I^{*}$.

ProOF. Suppose that $I \neq I^{*}$. Since $I^{*}$ is finitely generated, $I$ is contained in a maximal submodule $M$ of $I^{*}$, by [1, Theorem 2.8]. Thus $M^{*} \neq I^{*}$ by Theorem 4.4 However, $I^{*} \subseteq M^{*} \subseteq I^{*}$, a contradiction.

PROPOSITION 4.6. Let $R$ be a right $S F$ ring.

(1) If $J(M)=0$ for any flat right $R$-module $M$, then every simple right $R$-module is cotorsion.

(2) If $J(M)=0$ for any cyclic flat right $R$-module $M$, then $R$ is a right pure- $V$-ring.

PROOF. (1). Let $K$ be any pure submodule of any flat right $R$-module $N$, and $S$ any simple right $R$-module. It is enough to show that every homomorphism $f: K \rightarrow S$ 
can be extended to $M$. This is trivial if $f=0$. So we may assume $f \neq 0$. Since $S \cong K / \operatorname{ker}(f)$ and $S$ is flat, $\operatorname{ker}(f)$ is a pure submodule of $K$. Thus $\operatorname{ker}(f)$ is a pure submodule of $N$ since $K$ is pure in $N$. By hypothesis, $J(N / \operatorname{ker}(f))=0$, which implies that $\operatorname{ker}(f)$ is an intersection of maximal submodules of $N$. Let $x \in K$ and $x \notin \operatorname{ker}(f)$. Then there exists a maximal submodule $H$ of $N$ such that $\operatorname{ker}(f) \subseteq H$ and $x \notin H$. Therefore $N=H+K$. Note that $H \cap K=\operatorname{ker}(f)$, so we can extend $f$ to $g: N \rightarrow S$ by defining $g(h+k)=f(k)$ for any $h \in H$ and any $k \in K$.

(2) follows from the proof of (1).

\section{Acknowledgements}

This research was partially supported by the Specialized Research Fund for the Doctoral Program of Higher Education of China (No. 20050284015), NSF of China (No. 10331030) and the Nanjing Institute of Technology of China. It is the authors' pleasure to thank Professor Mohamed F. Yousif for several helpful conversations during The Fourth China-Japan-Korea International Symposium on Ring Theory and his visit to Nanjing University. The authors would also like to thank the referee for the comments and suggestions.

\section{References}

[1] F. W. Anderson and K. R. Fuller, Rings and Categories of Modules (Springer, Berlin, 1974).

[2] P. A. Guil Asensio and I. Herzog, 'Left cotorsion rings', Bull. London Math. Soc. 36 (2004), 303-309.

[3] —_, 'Sigma-cotorsion rings', Adv. Math. 191 (2005), 11-28.

[4] G. Azumaya, 'Finite splitness and finite projectivity', J. Algebra 106 (1987), 114-134.

[5] G. Baccella, 'On $\mathscr{C}$-semisimple rings. A study of the socle of a ring', Comm. Algebra 8 (1980), 889-909.

[6] L. Bican, E. Bashir and E. E. Enochs, 'All modules have flat covers', Bull. London Math. Soc. 33 (2001), 385-390.

[7] N. Q. Ding, 'On envelopes with the unique mapping property', Comm. Algebra 24 (1996), 14591470.

[8] N. V. Dung and P. F. Smith, 'On semi-artinian V-modules', J. Pure Appl. Algebra 82 (1992), 27-37.

[9] E. E. Enochs, 'Flat covers and flat cotorsion modules', Proc. Amer. Math. Soc. 92 (1984), 179-184.

[10] E. E. Enochs and O. M. G. Jenda, Relative Homological Algebra (Walter de Gruyter, Berlin, 2000).

[11] C. Faith, Lectures on Injective Modules and Quotient Rings, Lecture Notes in Math. 49 (Springer, Berlin, 1967).

[12] F. Kasch, Modules and Rings (Academic Press, London, 1982).

[13] H. Krause and M. Saorín, 'On minimal approximations of modules', Contemp. Math. 229 (1998), 227-236.

[14] T. Y. Lam, Lectures on Modules and Rings (Springer, Berlin, 1999).

[15] B. L. Lee, 'h-divisible modules', Comm. Algebra 31 (2003), 513-525. 
[16] L. X. Mao and N.Q. Ding, 'Notes on cotorsion modules', Comm. Algebra 33 (2005), 349-360.

[17] R. Yue Chi Ming, 'On V-rings and prime rings', J. Algebra 62 (1980), 13-20.

[18] W. K. Nicholson and J. F. Watters, 'Rings with projective socle', Proc. Amer. Math. Soc. 102 (1988), 443-450.

[19] W. K. Nicholson and M. F. Yousif, Quasi-Frobenius Rings, Cambridge Tracts in Math. 158 (Cambridge University Press, Cambridge, 2003).

[20] G. Puninski and P. Rothmaler, 'When every finitely generated flat module is projective', J. Algebra 277 (2004), 542-558.

[21] J. J. Rotman, An Introduction to Homological Algebra (Academic Press, New York, 1979).

[22] J. R. García Rozas and B. Torrecillas, 'Relative injective covers', Comm. Algebra 22 (1994), 2925-2940.

[23] P. F. Smith, 'Injective modules and prime ideals', Comm. Algebra 9 (1981), 989-999.

[24] N. M. A. Thani, 'Pure baer injective modules', Internat. J. Math. Math. Sci. 20 (1997), 529-538.

[25] J. Xu, Flat Covers of Modules, Lecture Notes in Math. 1634 (Springer, Berlin, 1996).

[26] S. L. Zhu, 'On rings over which every flat left module is finitely projective', J. Algebra 139 (1991), 311-321.

Department of Basic Courses

Nanjing Institute of Technology

Nanjing 210013

China

and

Department of Mathematics

Nanjing University

Nanjing 210093

China

e-mail: maolx2@hotmail.com
Department of Mathematics

Nanjing University

Nanjing 210093, China

e-mail: nqding@nju.edu.cn 
J. Aust. Math. Soc. 81 (2006) 